\title{
Adaptive Alternating Minimization Algorithms
}

Urs Niesen

\author{
Devavrat Shah \\ Massachusetts Institute of Technology \\ Department of Electrical Engineering and Computer Science \\ Cambridge, MA \\ Email: \{uniesen,devavrat,gww\}@mit.edu
}

\begin{abstract}
The classical alternating minimization (or projection) algorithm has been successful in the context of solving optimization problems over two variables or equivalently of finding a point in the intersection of two sets. The iterative nature and simplicity of the algorithm has led to its application to many areas such as signal processing, information theory, control, and finance.

A general set of sufficient conditions for the convergence and correctness of the algorithm is quite well-known when the underlying problem parameters are fixed. In many practical situations, however, the underlying problem parameters are changing over time, and the use of an adaptive algorithm is more appropriate. In this paper, we study such an adaptive version of the alternating minimization algorithm. As a main result of this paper, we provide a general set of sufficient conditions for the convergence and correctness of the adaptive algorithm. Perhaps surprisingly, these conditions seem to be the minimal ones one would expect in such an adaptive setting. Our result is a generalization of the work by Csiszár and Tusnády on alternating minimization procedures. We present applications of our results to adaptive decomposition of mixtures, adaptive logoptimal portfolio selection, and adaptive filter design.
\end{abstract}

\section{INTRODUCTION}

\section{A. Background}

The problem of finding a point in the intersection of two sets or equivalently of solving an optimization problem over two variables over a product space is central to many applications in areas such as signal processing, information theory, statistics, control, and finance. The alternating minimization or projection algorithm has been extensively used in such applications due to its iterative nature and simplicity.

The alternating minimization algorithm attempts to solve a minimization problem of the following form: given $\mathcal{P}, \mathcal{Q}$ and a function $D: \mathcal{P} \times \mathcal{Q} \rightarrow \mathbb{R}$, minimize $D$ over $\mathcal{P} \times \mathcal{Q}$. That is, find

$$
\min _{(P, Q) \in \mathcal{P} \times \mathcal{Q}} D(P, Q)
$$

Often minimizing over both variables simultaneously is not straightforward. However, minimizing with respect to one variable while keeping the other one fixed is often easy and sometimes possible analytically. In such a situation, the alternating minimization algorithm described next is well suited:

This work was supported in part by NSF under Grant No. CCF-0515109, and by HP through the MIT/HP Alliance. start with an arbitrary initial point $Q_{0} \in \mathcal{Q}$; for $n \geq 1$, iteratively compute

$$
\begin{aligned}
& P_{n} \in \underset{P \in \mathcal{P}}{\arg \min } D\left(P, Q_{n-1}\right), \\
& Q_{n} \in \underset{Q}{\arg \min } D\left(P_{n}, Q\right) .
\end{aligned}
$$

In other words, instead of solving the original minimization problem over two variables, the alternating minimization algorithm solves a sequence of minimization problems over only one variable. If the algorithm converges, the converged values are declared the solution to the original problem. Conditions for the convergence and correctness of such an algorithm, that is, conditions for

$$
\lim _{n \rightarrow \infty} D\left(P_{n}, Q_{n}\right)=\min _{(P, Q) \in \mathcal{P} \times \mathcal{Q}} D(P, Q),
$$

have been of interest since the early 1950s. A general set of conditions, stated in the paper by Csiszár and Tusnády [1], is summarized in the next theorem.

Theorem 1. Let $\mathcal{P}$ and $\mathcal{Q}$ be any two sets, and let $D$ : $\mathcal{P} \times \mathcal{Q} \rightarrow \mathbb{R} \cup\{+\infty\}$. Then the alternating minimization algorithm converges, i.e. (2) holds, if there exists $P \in \mathcal{P}$ such that $D\left(P, Q_{0}\right)<\infty$, and if there exists a nonnegative function $\delta: \mathcal{P} \times \mathcal{P} \rightarrow \mathbb{R}_{+} \cup\{+\infty\}$ such that for all $n \geq 1$ the following two properties hold:

(a) Three point property $\left(P, P_{n}, Q_{n-1}\right)$ :

$$
\delta\left(P, P_{n}\right)+D\left(P_{n}, Q_{n-1}\right) \leq D\left(P, Q_{n-1}\right), \quad \forall P \in \mathcal{P} .
$$

(b) Four point property $\left(P, Q, P_{n}, Q_{n}\right)$ :

$$
D\left(P, Q_{n}\right) \leq D(P, Q)+\delta\left(P, P_{n}\right), \quad \forall P \in \mathcal{P}, Q \in \mathcal{Q} .
$$

\section{B. Our Contribution}

In this paper, we consider an adaptive version of the above minimization problem. As before, suppose we wish to find

$$
\min _{(P, Q) \in \mathcal{P} \times \mathcal{Q}} D(P, Q)
$$

by means of an alternating minimization algorithm. However, on the $n$-th iteration, we are provided with sets $\mathcal{P}_{n}, \mathcal{Q}_{n}$ which are noisy versions of the sets $\mathcal{P}$ and $\mathcal{Q}$, respectively. That is, we are given a sequence of optimization problems

$$
\left\{\min _{(P, Q) \in \mathcal{P}_{n} \times \mathcal{Q}_{n}} D(P, Q)\right\}_{n \geq 0} .
$$


Such situations arise naturally in many applications. For example, in adaptive signal processing problems, the changing parameters could be caused by a slowly time-varying system, with the index $n$ representing time. An obvious approach is to solve each of the problems in (3) independently (one at each time instance $n$ ). However, since the system varies only slowly with time, such an approach is likely to result in redundant computation. Indeed, it is likely that a solution to the problem at time instance $n-1$ will be close to the one at time instance $n$. A different approach is to use an adaptive algorithm instead. Such an adaptive algorithm should be computationally efficient: given the tentative solution at time $n-1$, the tentative solution at time $n$ should be easy to compute. Moreover, if the time-varying system eventually reaches steady state, the algorithm should converge to the optimal steady state solution. In other words, instead of insisting that the adaptive algorithm solves (3) for every $n$, we only impose that it does so as $n \rightarrow \infty$.

Given these requirement, a natural candidate for such an algorithm is the following adaptation of the alternating minimization algorithm: choose an arbitrary initial $Q_{0} \in \mathcal{Q}_{0}$; for $n \geq 1$ compute (as in (1))

$$
\begin{aligned}
& P_{n} \in \underset{P \in \mathcal{P}_{n}}{\arg \min } D\left(P, Q_{n-1}\right), \\
& Q_{n} \in \underset{Q \in \mathcal{Q}}{\arg \min } D\left(P_{n}, Q\right) .
\end{aligned}
$$

Suppose that the sequences of sets $\left\{\mathcal{P}_{n}\right\}_{n \geq 0}$ and $\left\{\mathcal{Q}_{n}\right\}_{n \geq 0}$ converge (in a sense to be made precise later) to sets $\mathcal{P}$ and $\mathcal{Q}$, respectively. We are interested in conditions under which $D\left(P_{n}, Q_{n}\right)$ converges to

$$
\min _{(P, Q) \in \mathcal{P} \times \mathcal{Q}} D(P, Q)
$$

for large $n$. As a main result of this paper, we provide a general set of sufficient conditions under which this adaptive algorithm converges to the correct value. These conditions are essentially the same as those of [1] described in Theorem 1. The precise results are stated in Theorem 2.

This work was motivated by several applications in which the need for an adaptive alternating minimization algorithm arises. We present three such applications from the areas of estimation, finance, and signal processing.

\section{Organization}

The remainder of this paper is organized as follows. In Section II, we describe the setup and notation. Section III provides a convergence result for a fairly general class of adaptive alternating minimization algorithms. We specialize this result to adaptive minimization of divergences in Section IV, and to adaptive minimization procedures in Hilbert spaces (with respect to inner product induced norm) in Section V. We present an application in the divergence minimization setting from statistics and finance in Section IV, and an application in the Hilbert space setting from adaptive signal processing in Section V. Section VI contains concluding remarks.

\section{Notations AND Technical PReliminaries}

In this section, we setup notations and present technical preliminaries needed in the remainder of the paper. Let $(\mathcal{M}, d)$ be a compact metric space. Given two sets $\mathcal{A}, \mathcal{B} \subset \mathcal{M}$, define the Hausdorff distance between them as

$$
d_{H}(\mathcal{A}, \mathcal{B}) \triangleq \max \left\{\sup _{A \in \mathcal{A}} \inf _{B \in \mathcal{B}} d(A, B), \sup _{B \in \mathcal{B}} \inf _{A \in \mathcal{A}} d(A, B)\right\} .
$$

Consider a continuous function $D: \mathcal{M} \times \mathcal{M} \rightarrow \mathbb{R}$. For compact sets $\mathcal{A}, \mathcal{B} \subset \mathcal{M}$, define the set

$$
\mathcal{G}(\mathcal{A}, \mathcal{B}) \triangleq \underset{(A, B) \in \mathcal{A} \times \mathcal{B}}{\arg \min } D(A, B) .
$$

With slight abuse of notation, let

$$
D(\mathcal{A}, \mathcal{B}) \triangleq \min _{(A, B) \in \mathcal{A} \times \mathcal{B}} D(A, B) .
$$

Due to compactness of the sets $\mathcal{A}, \mathcal{B}$ and continuity of $D$, we have $\mathcal{G}(\mathcal{A}, \mathcal{B}) \neq \emptyset$, and hence $D(\mathcal{A}, \mathcal{B})$ is well-defined.

Let $(\mathcal{X}, d)$ be a metric space and $f: \mathcal{X} \rightarrow \mathbb{R}$. Define the modulus of continuity $\omega: \mathbb{R}_{+} \rightarrow \mathbb{R}_{+}$of $f$ as

$$
\omega(t) \triangleq \sup _{\substack{x, x^{\prime} \in \mathcal{X} \\ d\left(x, x^{\prime}\right) \leq t}}\left|f(x)-f\left(x^{\prime}\right)\right| .
$$

Remark 1 . Note that if $f$ is uniformly continuous then $w(t) \rightarrow$ 0 as $t \rightarrow 0$. In particular, this holds if $(\mathcal{X}, d)$ is compact and $f$ is continuous.

\section{Adaptive Alternating Minimization ALGORITHMS}

Here we present the precise problem setup. We then present an adaptive algorithm and sufficient conditions for its convergence and correctness.

\section{A. Setup}

Consider a compact metric space $(\mathcal{M}, d)$, compact sets $\mathcal{P}, \mathcal{Q} \subset \mathcal{M}$, and a continuous cost function $D: \mathcal{M} \times \mathcal{M} \rightarrow \mathbb{R}$. We want to find $D(\mathcal{P}, \mathcal{Q})$. However, we are not given the sets $\mathcal{P}, \mathcal{Q}$ directly. Instead, we are given a sequence of compact sets $\left\{\left(\mathcal{P}_{n}, \mathcal{Q}_{n}\right)\right\}_{n>0}: \mathcal{P}_{n}, \mathcal{Q}_{n} \subset \mathcal{M}$ are revealed at time $n$ such that as $n \rightarrow \infty, \mathcal{P}_{n} \stackrel{d_{H}}{\rightarrow} \mathcal{P}$ and $\mathcal{Q}_{n} \stackrel{d_{H}}{\rightarrow} \mathcal{Q}$. Given an arbitrary initial $\left(P_{0}, Q_{0}\right) \in \mathcal{P}_{0} \times \mathcal{Q}_{0}$, the goal is to find a sequence of points $\left(P_{n}, Q_{n}\right) \in \mathcal{P}_{n} \times \mathcal{Q}_{n}$ so that

$$
\lim _{n \rightarrow \infty} D\left(P_{n}, Q_{n}\right)=D(\mathcal{P}, \mathcal{Q}) \text {. }
$$

\section{B. Algorithm}

The setup described in the last section suggests the following adaptive version of the alternating minimization algorithm for the above setup. Initially, we have $\left(P_{0}, Q_{0}\right) \in \mathcal{P}_{0} \times \mathcal{Q}_{0}$. Define recursively: for $n \geq 1$, pick any

$$
\begin{aligned}
& P_{n} \in \underset{P \in \mathcal{P}_{n}}{\arg \min } D\left(P, Q_{n-1}\right), \\
& Q_{n} \in \underset{Q \in \mathcal{Q}_{n}}{\arg \min } D\left(P_{n}, Q\right) .
\end{aligned}
$$

We call this the AAM (Adaptive Alternating Minimization) algorithm in the following. Note that if $\mathcal{P}_{n}=\mathcal{P}$ and $\mathcal{Q}_{n}=\mathcal{Q}$ for all $n$, then the above algorithm is the same as the classical alternating minimization algorithm. 


\section{Sufficient Conditions for Convergence}

In this section, we present a set of sufficient conditions under which the AAM algorithm converges to $D(\mathcal{P}, \mathcal{Q})$. As we shall see, we need "three point" and "four point" properties (equivalent to those in [1]) also in the adaptive setup. To this end, assume there exists a continuous function $\delta: \mathcal{M} \times \mathcal{M} \rightarrow$ $\mathbb{R}$ such that the following conditions are satisfied.

C1. Three point property $\left(P, P_{n}, Q_{n-1}\right)$ : for all $n \geq 1$,

$$
\delta\left(P, P_{n}\right)+D\left(P_{n}, Q_{n-1}\right) \leq D\left(P, Q_{n-1}\right), \quad \forall P \in \mathcal{P}_{n} .
$$

C2. Four point property $\left(P, Q, P_{n}, Q_{n}\right)$ : for all $n \geq 1$,

$$
D\left(P, Q_{n}\right) \leq D(P, Q)+\delta\left(P, P_{n}\right), \quad \forall P \in \mathcal{P}_{n}, Q \in \mathcal{Q}_{n} .
$$

We are now ready to show convergence and correctness of the AAM algorithm.

Theorem 2. Let $\left\{\left(\mathcal{P}_{n}, \mathcal{Q}_{n}\right)\right\}_{n \geq 0}$ be compact subsets of $\mathcal{M}$ such that

$$
\mathcal{P}_{n} \stackrel{d_{H}}{\rightarrow} \mathcal{P}, \quad \mathcal{Q}_{n} \stackrel{d_{H}}{\rightarrow} \mathcal{Q},
$$

and let $D: \mathcal{M} \times \mathcal{M} \rightarrow \mathbb{R}$ be a continuous function. Let conditions $\mathrm{C} 1$ and $\mathrm{C} 2$ hold. Then, under the AAM algorithm,

$$
\liminf _{n \rightarrow \infty} D\left(P_{n}, Q_{n}\right)=D(\mathcal{P}, \mathcal{Q}),
$$

and all limit points of subsequences of $\left\{\left(P_{n}, Q_{n}\right)\right\}_{n>0}$ achieving this lim inf belong to $\mathcal{G}(\mathcal{P}, \mathcal{Q})$. If, in addition,

$$
\sum_{n=0}^{\infty} \omega\left(2 \varepsilon_{n}\right)<\infty
$$

where $\varepsilon_{n} \triangleq d_{H}\left(\mathcal{P}_{n}, \mathcal{P}\right)+d_{H}\left(\mathcal{Q}_{n}, \mathcal{Q}\right)$, and $\omega$ is the modulus of continuity of $D$, then

$$
\lim _{n \rightarrow \infty} D\left(P_{n}, Q_{n}\right)=D(\mathcal{P}, \mathcal{Q}),
$$

and all limit points of $\left\{\left(P_{n}, Q_{n}\right)\right\}_{n \geq 0}$ belong to $\mathcal{G}(\mathcal{P}, \mathcal{Q})$.

The proof of Theorem 2 can be found in [2].

Remark 2. Compared to the conditions of [1], the only additional requirement here is in essence uniform continuity of the function $D$ (which is implied by compactness of $\mathcal{M}$ and continuity of $D$ ), and summability of the $\omega\left(2 \varepsilon_{n}\right)$. This is the least one would expect in this adaptive setup to obtain a conclusion as in Theorem 2.

\section{Divergence Minimization}

In this section, we specialize the setup and algorithm from Section III to the special case of alternating divergence minimization. A large class of problems can be formulated as a minimization of divergences. For example, computation of channel capacity and rate distortion function [3], [4], selection of log-optimal portfolios [5], and maximum likelihood estimation from incomplete data [6]. These problems were shown to be divergence minimization problems in [1]. For further applications of alternating divergence minimization algorithms, see [7]. We describe applications to the problem of adaptive mixture decomposition and of adaptive log-optimal portfolio selection.

\section{A. Setup}

Given a finite set $\Sigma$ and some constant $0<\theta<\Theta$, let $\mathcal{M}=\mathcal{M}(\Sigma, \theta, \Theta)$ be the set of all measures $P$ on $\Sigma$ such that

$$
\sum_{\sigma \in \Sigma} P(\sigma) \leq \Theta, \text { and } P(\sigma) \geq \theta, \forall \sigma \in \Sigma .
$$

Endow $\mathcal{M}$ with the topology induced by the metric $d: \mathcal{M} \times$ $\mathcal{M} \rightarrow \mathbb{R}_{+}$defined as

$$
d(P, Q) \triangleq \max _{\sigma \in \Sigma}|P(\sigma)-Q(\sigma)| .
$$

It is easy to check that the metric space $(\mathcal{M}, d)$ is compact. The cost function $D$ of interest is divergence

$$
D(P, Q) \triangleq D(P \| Q) \triangleq \sum_{\sigma \in \Sigma} P(\sigma) \log \frac{P(\sigma)}{Q(\sigma)}
$$

for any $P, Q \in \mathcal{M}$. Note that (4) ensures that $D$ is well defined (i.e., does not take the value $\infty$ ). It is well-known (and easy to check) that the function $D$ is continuous and convex in both arguments. Finally, define the function $\delta$

$$
\delta(P, Q) \triangleq D(P \| Q)-\sum_{\sigma \in \Sigma}(P(\sigma)-Q(\sigma))
$$

for any $P, Q \in \mathcal{M}$.

In [1], it has been established that for convex $\mathcal{P}$ and $\mathcal{Q}$ the pair of functions $D, \delta$ satisfy $\mathrm{C} 1$ and $\mathrm{C} 2$. As stated above, the space $\mathcal{M}=\mathcal{M}(\Sigma, \theta, \Theta)$ with metric $d$ is a compact metric space, and the function $D$ is continuous. Hence Theorem 2 applies.

\section{B. Application: Decomposition of Mixtures and Log-Optimal Portfolio Selection}

We consider an application of our adaptive divergence minimization algorithm to the problem of decomposing a mixture. A special case of this setting yields the problem of log-optimal portfolio selection.

We are given a sequence of i.i.d. random variables $\left\{Y_{l}\right\}_{l \geq 0}$, each taking values in the finite set $\mathcal{Y} . Y_{l}$ is distributed according to the mixture $\sum_{i=1}^{I} c_{i} \mu_{i}$, where the $\left\{c_{i}\right\}_{i=1}^{I}$ sum to one, and $c_{i} \geq c_{0}>0$ for all $i \in\{1, \ldots, I\}$, and where $\left\{\mu_{i}\right\}_{i=1}^{I}$ are distributions on $\mathcal{Y}$. We assume that $\mu_{i}(y) \geq \mu_{0}>0$ for all $y \in \mathcal{Y}, i \in\{1, \ldots, I\}$. The goal is to compute an estimate of $\left\{c_{i}\right\}_{i=1}^{I}$ from $\left\{Y_{l}\right\}_{l=1}^{n}$ and knowing $\left\{\mu_{i}\right\}_{i=1}^{I}$.

Let $\bar{P}_{n}$ be the empirical distribution of $\left\{Y_{l}\right\}_{l=1}^{n}$. The maximum likelihood estimator of $\left\{c_{i}\right\}_{i=1}^{I}$ is given by (see, e.g., [8, Lemma 3.1])

$$
\underset{\left\{\tilde{c}_{i}\right\}}{\arg \min } D\left(\bar{P}_{n} \| \sum_{i=1}^{I} \tilde{c}_{i} \mu_{i}\right),
$$

Following [8, Example 5.1], we define

$$
\begin{aligned}
& \Sigma \triangleq\{1, \ldots, I\} \times \mathcal{Y}, \\
& \mathcal{Q}_{n}=\mathcal{Q} \triangleq\left\{Q: Q(i, y)=\tilde{c}_{i} \mu_{i}(y),\right. \\
&\left.\quad \quad \text { for some }\left\{\tilde{c}_{i}\right\} \text { with } \sum_{i} \tilde{c}_{i}=1, \tilde{c}_{i} \geq c_{0} \forall i\right\} \\
& \mathcal{P}_{n} \triangleq\left\{P: \sum_{i=1}^{I} P(i, y)=\bar{P}_{n}(y), P(i, y) \geq 0 \forall i, y\right\} .
\end{aligned}
$$


Note that $\mathcal{P}_{n}$ and $\mathcal{Q}$ are convex and compact. From [8, Lemma 5.1], we have

$$
\min _{\left\{\tilde{c}_{i}\right\}} D\left(\bar{P}_{n} \| \sum_{i=1}^{I} \tilde{c}_{i} \mu_{i}\right)=\min _{P \in \mathcal{P}_{n}} \min _{Q \in \mathcal{Q}} D(P \| Q),
$$

and the minimizer of the left hand side can be recovered from the corresponding marginal of the optimal $Q$ on the right hand side.

Fix a $P$, assuming without loss of generality that

$$
\sum_{y \in \mathcal{Y}} P(1, y) \geq \sum_{y \in \mathcal{Y}} P(2, y) \geq \ldots \geq \sum_{y \in \mathcal{Y}} P(I, y) .
$$

The $\left\{\tilde{c}_{i}\right\}$ minimizing $D(P \| Q)$ can be shown to be of the form $\tilde{c}_{i}>c_{0}$ for all $i \leq J^{*}$ and $\tilde{c}_{i}=c_{0}$ for all $i>J^{*}$. More precisely, define

$$
\eta(J) \triangleq \frac{1}{1-(I-J) c_{0}} \sum_{i=1}^{J} \sum_{y \in \mathcal{Y}} P(i, y),
$$

and choose $J^{*} \in\{1, \ldots, I+1\}$ such that

$$
\begin{gathered}
\frac{1}{\eta\left(J^{*}\right)} \sum_{y \in \mathcal{Y}} P\left(J^{*}, y\right)>c_{0}, \\
\frac{1}{\eta\left(J^{*}\right)} \sum_{y \in \mathcal{Y}} P\left(J^{*}+1, y\right) \leq c_{0},
\end{gathered}
$$

where $P(I+1, y) \triangleq 0$. Then the optimal $\left\{\tilde{c}_{i}\right\}$ are given by

$$
\tilde{c}_{i}=\frac{1}{\eta\left(J^{*}\right)} \sum_{y \in \mathcal{Y}} P(i, y)
$$

for $i \leq J^{*}$ and $\tilde{c}_{i}=c_{0}$ for $J^{*}<i \leq I$. For fixed $Q$, the minimizing $P$ is

$$
P(i, y)=\frac{\tilde{c}_{i} \mu_{i}(y)}{\sum_{i} \tilde{c}_{i} \mu_{i}(y)} \bar{P}_{n}(y) .
$$

We now check that (4) is satisfied. As $\mathcal{P}_{n}$ and $\mathcal{Q}$ are sets of distributions, we can choose $\Theta=1$. For all $Q \in \mathcal{Q}, i \in$ $\{1, \ldots, I\}, y \in \mathcal{Y}$, we have $Q(i, y) \geq \mu_{0} c_{0}>0$. However, for $P \in \mathcal{P}_{n}$, we have in general only $P(i, y) \geq 0$. In order to apply Theorem 2, we need to show that we can, without loss of optimality, restrict the sets $\mathcal{P}_{n}$ to contain only distributions $P$ that are bounded below by some $p_{0}>0$. In other words, we need to show that the projections on $\mathcal{P}_{n}$ are bounded below by $p_{0}$.

Assume for the moment that the empirical distribution $\bar{P}_{n}$ is close to the true one in the sense that

$$
\left|\bar{P}_{n}(y)-\sum_{i} c_{i} \mu_{i}(y)\right| \leq \frac{\mu_{0}}{2}
$$

for all $y \in \mathcal{Y}$. As $\sum_{i} c_{i} \mu_{i}(y) \geq \mu_{0}$ this implies $\bar{P}_{n}(y) \geq \frac{\mu_{0}}{2}$ for all $y$. From (6), this implies that the projection $P$ on $\mathcal{P}$ of any point in $\mathcal{Q}$ satisfies $P(i, y) \geq \frac{1}{2} c_{0} \mu_{0}^{2} \triangleq p_{0}$ for all $i \in\{1, \ldots, I\}, y \in \mathcal{Y}$. Hence $\mathcal{M}(\Sigma, \theta, \Theta)$ satisfies (4) with $\theta=\frac{1}{2} c_{0} \mu_{0}^{2}$ and $\Theta=1$.
It remains to argue that $\bar{P}_{n}$ is close to $\sum_{i} c_{i} \mu_{i}(y)$. Suppose instead of constructing the set $\mathcal{P}_{n}$ (see (5)) with respect to $\bar{P}_{n}$, we construct it with respect to the distribution $\overline{\bar{P}}_{n}$ defined as

$$
\overline{\bar{P}}_{n}(y) \triangleq \frac{\mu_{0}}{2}+\lambda\left(\bar{P}_{n}(y)-\frac{\mu_{0}}{2}\right)^{+},
$$

where $\lambda$ is chosen such that $\sum_{y} \bar{P}_{n}(y)=1$. $\bar{P}_{n}$ is bounded below by $\frac{\mu_{0}}{2}$ by construction. Moreover, by the strong law of large numbers,

$$
\mathbb{P}\left(\bar{P}_{n} \neq \bar{P}_{n} \text { i.o. }\right)=0 .
$$

Hence we have $\mathcal{P}_{n} \stackrel{d_{H}}{\rightarrow} \mathcal{P}$ almost surely, where $\mathcal{P}$ is constructed as in (5) with respect to the true distribution $\sum_{i} c_{i} \mu_{i}$.

Applying now Theorem 2 yields that under the AAM algorithm

$$
\liminf _{n \rightarrow \infty} D\left(P_{n}, Q_{n}\right)=D(\mathcal{P}, \mathcal{Q})
$$

almost surely, and that every limit point of $\left\{\left(P_{n}, Q_{n}\right)\right\}_{n \geq 0}$ achieving this lim inf is an element of $\mathcal{G}(\mathcal{P}, \mathcal{Q})$.

Since by the law of the iterated logarithm, convergence of $\bar{P}_{n}$ to $P$ is only $\Theta(\sqrt{\log \log n} / \sqrt{n})$ as $n \rightarrow \infty$ almost surely, and since $\omega(\varepsilon)=o(\varepsilon)$ as $\varepsilon \rightarrow 0$ only if $D$ is a constant [9, Chapter 2.6], we can in this scenario not conclude from Theorem 2 that $\lim _{n \rightarrow \infty} D\left(P_{n}, Q_{n}\right)=D(\mathcal{P}, \mathcal{Q})$.

As noted in [8], a special case of the decomposition of mixture problem is that of maximizing the expected value of $\log \sum_{i} c_{i} W_{i}$, where $\left\{W_{i}\right\}_{i=1}^{I}$ is distributed according to $\bar{P}_{n}$. The standard alternating divergence minimization algorithm is then the same as Cover's portfolio optimization algorithm [5]. Thus the AAM algorithm applied as before yields also an adaptive version of this portfolio optimization algorithm.

\section{Projections in Hilbert Space}

In this section, we specialize the setup and algorithm from Section III to the special case of minimization in a Hilbert space. A large class of problems can be formulated as alternating projections in Hilbert spaces. For example problems in filter design, signal recovery, and spectral estimation. For an extensive overview, see [10]. In the context of Hilbert spaces, the alternating minimization algorithm is often called POCS (Projection Onto Convex Sets).

\section{A. Setup}

Let $\mathcal{M}$ be a compact subset of a Hilbert space with the usual norm $d(A, B)^{2} \triangleq\langle A-B, A-B\rangle$. Then $(\mathcal{M}, d)$ is a compact metric space. The cost function $D$ of interest is

$$
D(A, B) \triangleq d(A, B)^{2} .
$$

The function $D$ is continuous, convex and nonnegative. Define the function $\delta$ (as part of conditions $\mathrm{C} 1$ and $\mathrm{C} 2$ ), as

$$
\delta(A, B) \triangleq d(A, B)^{2} .
$$

In [1], it has been established that for convex $\mathcal{P}$ and $\mathcal{Q}$ the pair of functions $D, \delta$ satisfies conditions $\mathrm{C} 1$ and $\mathrm{C} 2$ As stated above, the space $(\mathcal{M}, d)$ is a compact metric space, and the function $D$ is continuous. Hence Theorem 2 applies. 


\section{B. Application: Set Theoretic Signal Processing and Adaptive Filter Design}

In this section, we consider a problem in the Hilbert space setting as defined in Section V-A. Let $\left\{\mathcal{S}_{i}\right\}_{i=1}^{I}$ be a collection of convex compact subsets of the Hilbert space $\mathbb{R}^{k}$ with the usual inner product, and let $\left\{c_{i}\right\}_{i=1}^{I}$ be positive weights summing to one. In set theoretic signal processing, the objective is to find a point $A^{*}$ minimizing

$$
\sum_{i=1}^{I} c_{i} d\left(A, \mathcal{S}_{i}\right),
$$

where $d\left(A, \mathcal{S}_{i}\right) \triangleq \min _{S \in \mathcal{S}_{i}} d(A, S)$. Many problems in signal processing can be formulated in this way. Applications can be found for example in control, filter design, and estimation. For an overview and extensive list of references, see [10]. As an example, in a filter design problem, the $\mathcal{S}_{i}$ could be constraints on the impulse and frequency responses of a filter [11], [12].

Following [13], this problem can be formulated in our framework by defining the Hilbert space $\mathcal{H}=\mathbb{R}^{I k}$ with inner product

$$
\langle A, B\rangle \triangleq \sum_{i=1}^{I} c_{i}\left\langle A_{i}, B_{i}\right\rangle,
$$

where $A_{i}, B_{i} \in \mathbb{R}^{k}$ for $i \in\{1, \ldots, I\}$ are the components of $A$ and $B$. Let

$$
\mathcal{S} \triangleq \operatorname{conv}\left\{\cup_{i=1}^{I} \mathcal{S}_{i}\right\} \subset \mathbb{R}^{k}
$$

and let

$$
\mathcal{M} \triangleq \mathcal{S}^{I} \subset \mathcal{H}
$$

be the $I$-fold product of the convex hull of the constraint sets $\left\{\mathcal{S}_{i}\right\}_{i=1}^{I}$. Since each of the sets $\mathcal{S}_{i}$ is bounded, $\mathcal{M}$ is bounded and by definition also convex and closed. We define the set $\mathcal{P} \subset \mathcal{M}$ as

$$
\mathcal{P} \triangleq\{(\widetilde{P}, \ldots, \widetilde{P}) \in \mathcal{H}: \widetilde{P} \in \mathcal{S}\}
$$

and the set $\mathcal{Q} \subset \mathcal{M}$ as

$$
\mathcal{Q} \triangleq \mathcal{S}_{1} \times \cdots \times \mathcal{S}_{I} .
$$

For a fixed $P \in \mathcal{P}$, the $Q \in \mathcal{Q}$ minimizing $D(P, Q)$ has the form

$$
\left(S_{1}(\widetilde{P}), \ldots, S_{I}(\widetilde{P})\right),
$$

where $S_{i}(\widetilde{P})$ is the $Q_{i} \in \mathcal{S}_{i}$ minimizing $\left\|\widetilde{P}-\widetilde{Q}_{i}\right\|^{2}$. For a fixed $Q=\left(Q_{1}, \ldots, Q_{I}\right) \in \mathcal{Q}$ the $P \in \mathcal{P}$ minimizing $D(P, Q)$ is given by

$$
\left(\sum_{i=1}^{I} c_{i} Q_{i}, \ldots, \sum_{i=1}^{I} c_{i} Q_{i}\right) .
$$

Moreover, a solution to (7) can be found from the standard alternating minimization algorithm for Hilbert spaces on $\mathcal{P}$ and $\mathcal{Q}$.

Up to this point, we have assumed that the constraint sets $\left\{\mathcal{S}_{i}\right\}_{i=1}^{I}$ are constant. The results from Section III, enable us to look at situations in which the constraint sets $\left\{\mathcal{S}_{i, n}\right\}_{i=1}^{I}$ are time-varying. Coming back to the filter design example mentioned above, we are now interested in an adaptive filter. The need for such filters arises in many different situations (see, e.g., [14]).

The time-varying sets $\left\{\mathcal{S}_{i, n}\right\}_{i=1}^{I}$ give rise to sets $\mathcal{Q}_{n}$, defined in analogy to (8). We assume again that $\mathcal{S}_{i, n} \stackrel{d_{H}}{\longrightarrow} \mathcal{S}_{i}$ for all $i \in\{1, \ldots, I\}$, and let $\mathcal{Q}$ be defined with respect to the limiting $\left\{S_{i}\right\}_{i=1}^{I}$ as before. Applying Theorem 2, we obtain that under the AAM algorithm $\liminf _{n \rightarrow \infty} D\left(P_{n}, Q_{n}\right)=D(\mathcal{P}, \mathcal{Q})$, every limit point of subsequences of $\left\{\left(P_{n}, Q_{n}\right)\right\}_{n}>0$ achieving this lim inf is in $\mathcal{G}(\mathcal{P}, \mathcal{Q})$, and if also $\sum_{n=0}^{\infty} \omega\left(2 \varepsilon_{n}\right)<\infty$ then $\lim _{n \rightarrow \infty} D\left(P_{n}, Q_{n}\right)=D(\mathcal{P}, \mathcal{Q})$, and every limit point of $\left\{\left(P_{n}, Q_{n}\right)\right\}_{n \geq 0}$ is in $\mathcal{G}(\mathcal{P}, \mathcal{Q})$.

\section{CONCLUSIONS}

We considered a fairly general adaptive alternating minimization algorithm, and found sufficient conditions for its convergence and correctness. This adaptive algorithm has applications in a variety of settings. We discussed in detail how to apply it to three different problems (from statistics, finance, and signal processing).

\section{REFERENCES}

[1] I. Csiszár and G. Tusnády. Information geometry and alternating minimization procedures. Statistics \& Decisions, Supplement Issue, (1):205-237, 1984 .

[2] U. Niesen, D. Shah, and G. Wornell. Adaptive alternating minimization algorithms. submitted to the IEEE Transactions on Information Theory, December 2006.

[3] S. Arimoto. An algorithm for computing the capacity of arbitrary discrete memoryless channels. IEEE Transactions on Information Theory, 18(1):14-20, January 1972.

[4] R. E. Blahut. Computation of channel capacity and rate distortion functions. IEEE Transactions on Information Theory, 18(4):460-473, July 1972 .

[5] T. M. Cover. An algorithm for maximizing expected log investment return. IEEE Transactions on Information Theory, 30(2):369-373, March 1984.

[6] A. P. Dempster, N. M. Laird, and D. B. Rubin. Maximum likelihood from incomplete data via the EM algorithm. Joumal of the Royal Statistical Society, Series B, 39(1):1-38, November 1977.

[7] J. A. O'Sullivan. Alternating minimization algorithms: From BlahutArimoto to expecation-maximization. In A. Vardy, editor, Codes, Curves, and Signals: Common Threads in Communications, pages 173-192. Kluwer Academic, 1998.

[8] I. Csiszár and P. C. Shields. Information Theory and Statistics: A Tutorial. Now Publishers, 2004.

[9] R. A. DeVore and G. G. Lorentz. Constructive Approximation. Springer, 1993.

[10] P. L. Combettes. The foundations of set theoretic estimation. Proceedings of the IEEE, 81(2):182-208, February 1993.

[11] A. E. Çetin, Ö. N. Gerek, and Y. Yardimci. Equiripple FIR filter design by the FFT algorithm. IEEE Signal Processing Magazine, 14(2):60-64, March 1997.

[12] R. A. Nobakht and M. R. Civanlar. Optimal pulse shape design for digital communication systems by projections onto convex sets. IEEE Transactions on Communications, 43(12):2874-2877, December 1995.

[13] P. L. Combettes. Inconsistent signal feasibility problems: Least square solutions in a product space. IEEE Transactions on Signal Processing, 42(11):2955-2966, November 1994.

[14] S. Haykin. Adaptive Filter Theory. Prentice-Hall, 1996. 